\title{
Modified artificial bee colony optimization algorithm for adaptive power scheduling in an isolated system
}

\author{
Vijo M Joy, S Krishnakumar \\ School of Technology and Applied Sciences, M G University Research Centre, Kerala, India
}

\begin{tabular}{l}
\hline \hline Article Info \\
\hline Article history: \\
Received May 15, 2020 \\
Revised Jul 23, 2020 \\
Accepted Aug 7, 2020 \\
\hline
\end{tabular}

\section{Keywords:}

Artificial bee colony

Artificial neural network

Backpropagation

Optimization

Power scheduling

\begin{abstract}
The objective of this work is to solve the power scheduling problems for efficient energy management by assigning the optimal values. Artificial neural networks are used widely in the field of energy management and load scheduling. The backpropagation technique is used for the feed-forward neural network training and the Levenberg-Marquardt algorithm is used to minimize the errors. The slow speed of convergence and getting stuck in local minima are some negatives of backpropagation in complex computation. To overcome these drawbacks an innovative meta-heuristic search algorithm called modified artificial bee colony optimization algorithm is used. a hybrid neural network is introduced in this work. The simulation result shows that the efficiency of the system is improved when hybrid optimization is used. With this method, the system achieves an optimal accuracy of $99.23 \%$.
\end{abstract}

This is an open access article under the CC BY-SA license.

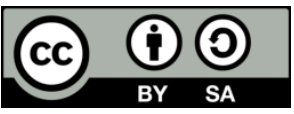

Corresponding Author:

Vijo M Joy

School of Technology and Applied Sciences

Research Centre Mahatma Gandhi University

Edappally, Cochin- 24, Kerala, India

Email: vijomjoy@gmail.com

\section{INTRODUCTION}

In an interrelated power system, the load scheduling strategy is concerned with accurate scheduling of power based on the parameters like availability, expense, and on-demand. Power generation from the nonconventional energy sources is less expensive when compared with other power sources like hydroelectric, thermal, and nuclear. In this, many parameters are considered for the generation of power from each source [1]. The intensity of light is one of the major parameters that affect the response of the solar system. The hydropower station mainly depends on the availability of water. That is, both power stations are affected by weather conditions. In a thermal power station, it is not affected by the weather conditions, but its production cost is high. In the demand side, many parameters are considered for scheduling $[2,3]$. Type of use, power usage area, cost, population, temperature, and priority-based allotment are the main parameters considered in the demand side scheduling [4]. Artificial neural network (ANN) is the best approach in machine learning algorithms [5]. It designing and modeling is based on the human brain concept. Depending on the structure and training model, there are many types of ANNs [6]. Here, backpropagation (BP) is assumed as the most powerful error reducing technique. A hybridized algorithm - Artificial Bee Colony- Backpropagation (ABCBP) - is adopted for the enhanced result.

In India, as in the year 2019, the gross installed capacity of the power system is 35.61 million kilowatts. In this, $64 \%$ made up of thermal power $14 \%$ from hydropower, a small part of nuclear power, and remaining from other renewable power sources. Nowadays, the importance of optimal power scheduling is a major concern in the research field. The optimal power scheduling ensures the power based on cost, 
availability, and demand. Modern and intellectual solutions are developed in recent years, such as the genetic algorithm and artificial neural network, which have high accuracy when compared with the traditional methods. To solve the different optimization problems various optimization techniques based on population are used. Being the collective intelligent behavior, the swarm intelligence-related algorithms achieved the creditable result. When searching for a better source of food, the real bees have uniform nature. Mouti and Hawary [7] proposed an ABC algorithm based on the identical behavior of bees. MATLAB is used for the simulation.

In the past few decades, a number of researchers engaged in studying the scheduling problem. Hota, et al. [8] proposed an efficient short-term hydrothermal scheduling algorithm based on the evolutionary programming technique. Sifuentes et al. [9] developed a Lagrangian relaxation-based optimization algorithm to detect the ideal solution in hydrothermal scheduling. Basu [10] highlights a simple and effective approach to thermal plant scheduling with hydroelectric units. In that work optimization related neural network formulation is used to determine the hydrothermal scheduling. A method - differential evolution - is proposed by Le et al. [11] to handle the dispatch problems in the hydrothermal power system. In that work, they aim to reduce electricity generation costs. Chiang [12] proposed an enhanced genetic optimization algorithm towards the optimal cost-effective emission dispatch of the hydrothermal power system. Rongrong et.al. [13] suggested a model in the power market for a hydro-thermal-nuclear power system. A two-stage design method for an efficient power dispatching is introduced by Chen et al. [14] and they control the coordination parameters in $\mathrm{ABC}$ algorithm for economic power scheduling. Wong [15] proposed a hybrid optimization technique for a short term hydrothermal scheduling. In that work, they present an optimization technique based on the population to solve different optimization problems. The real bees are maintaining swarm intelligence behavior when searching for food sources with good quality. Based on this behavior ABC algorithm is developed to explain different problems [16]. Lenin et al. [17] introduced a Hybrid Biogeography method in a power system for solving the multi-objective reactive power dispatch problem.

\section{RESEARCH METHOD}

\subsection{Backpropagation neural network}

The BP Neural Network (BPNN) is a powerful technique based on supervised learning ANN to distinguish the interruption activities of the system. The neuron is the basic element of this technique used to process the information from its memory $[18,19]$. The error-correction procedure is used by BPNN to learn. This method minimizes the error by modifying the connection weights. To reduce the error rate, tune the weights in a proper manner, and to create the model predictable by increasing its generalization. The error function is shown in (1) and it is the difference between the actual and anticipated values [20].

$$
\begin{aligned}
& e_{i}=\left(t_{i}-o_{i}\right) \\
& E=\frac{1}{2} \sum_{i=0}^{j}\left(t_{i}-o_{i}\right)^{2}
\end{aligned}
$$

Where $i$ is the index of layer, $t_{i}$ expected response is and $O_{\mathrm{i}}$ is the actual response of the network. There are three layers in a typical BPNN; input layer, an output layer, and a hidden layer. If the significant of classifications exceed the limit $(>20)$ then the hidden layer should be increased to two or more. The BPNN technique is summarized as follows: (a) computation using feed-forward NN (b) backward propagation of the error to the hidden layer and output layer, (c) bring up to date the connection weights. The weight modifying process can be expressed as follows

$$
\Delta w_{j i}=-\eta \frac{\partial \boldsymbol{E}}{\partial w_{j i}}, w_{j i}^{\prime}=w_{j i}+\Delta w_{j i}
$$

Where $i$ and $j$ are the index of layers, $\Delta w_{j i}$ is the updates of weight vectors for the network connections; $\eta$ is the learning rate constant and $w_{j i}^{\prime}$ is the sum of recent and updated connection weights between the layers [21]. The algorithm stops its refining process when the error function value becomes less than the standard value [22]. For the better stabilization, the Levenberg- Marquardt optimization technique is 
used in the BPNN. BP is considered as a suitable algorithm in the multi-layer perceptron [23]. The feedforward ANN structure is learned using the BP algorithm with the constraints as shown in Table 1.

\begin{tabular}{cc}
\multicolumn{2}{c}{ Table 1. ANN parameters } \\
\hline Parameter & Value \\
\hline Threshold range & {$[-1,1]$} \\
Learning coefficient & 0.2 \\
Momentum & 0.8 \\
Activation function & Sigmoid \\
Weight range & {$[-1,1]$} \\
Stopping rule & 1000 \\
\hline
\end{tabular}

Nowadays, it is the ongoing leading developmental algorithms due to its optimistic features like flexibility, simple in concept, and implementation [24]. To solve different optimization and engineering complications, this algorithm is successfully applied. The intelligent searching behavior of bees is simulated complications, this algorithm is successfully applied. The intelligent searching behavior of bees is simulated for the optimization problems [25]. These algorithms mainly have employed bees, scout bees, onlooker bees, and food sources. The effective teamwork between these bees can examine the best sources of food.

An employed bee updates the source position in her memory and tracks down a new food source position. They analyze the nectar amount of the new food source with existing and memorize the best one. They come back to the hive and dancing with others to share the information regarding nectar amounts. Dance duration depends on the nectar amount of food sources. Onlooker bees determine a good quality food source by watching the dances of employed bees [26]. The same employed bee becomes a scout when the source is abandoned and restarts to search for different food sources randomly [27]. In the present algorithm, the number of employed bees is equal to the number of food sources [28]. Similarly, the number of employed bees and onlooker bees are the same. That is, fifty percent of the bee colony is occupied by an employed bee. Algorithm 1:

a) Population Initialization

b) Distribute employed bees for a new food source

c) Store the best source of food by evaluating the fitness

d) Development of Onlooker bees

e) Calculate Probabilities for Onlookers

f) Development of Scout Bees

g) Remember the best-realized solution so far

h) Stop the procedure when the unit cycle equal to Maximum Cycle Number

i) Otherwise, go back to Step 2

This algorithm, by using (1), randomly determines the food sources [29].

$$
x_{i j}=x_{\min , j}+\operatorname{rand}[0,1]\left(x_{\max , j}-x_{\min , j}\right)
$$

Where $i$ represents the food source and $j$ represents the constraint to be optimized, $x_{\min }$ and $x_{\max }$ are lower and upper boundary parameters for the solution respectively, and rand $[0,1]$ creates arbitrary significant values among 0 and 1 . A possible solution is generated by the employed bees by updating the (4) using the expression

$$
v_{i j}=x_{i j}+\operatorname{rand}[-1,+1]\left(x_{i j}-x_{k j}\right)
$$

Where $k$ and $j$ are randomly selected indices that denote the food source and optimization parameters respectively and $\operatorname{rand}[-1,+1]$ is a random number that controls the neighbor food source production. The fitness of the optimum solution is evaluated by the employed bees and the new solution is to replace the existence in the memory of employed bees. The bee remains its food source if the nectar amount of the recent source of food is lesser than that of the current one.

Using the probability comparison method, the onlooker bees choose their sources of food. The probability function $P_{i}$ is calculated using the fitness value. The nectar sources of good probability are carefully chosen and the probability of being selected for the food sources is calculated as follows: 


$$
P_{i}=\frac{f i t_{i}}{\sum_{n=1}^{S N} f i t_{n}}
$$

Where, fitness or profitability (quality of the possible solution) value of the $i^{\text {th }}$ solution is $\mathrm{fit}_{i}$, which is derived from the (7) and $\mathrm{SN}$, is the employed or onlooker bees size.

$$
f i t_{i}= \begin{cases}\frac{1}{1+f_{i}} & f_{i} \geq 0 \\ 1+\left|f_{i}\right| & f \angle 0\end{cases}
$$

Where, independent function value for $i$ is $f_{i}$, and it is distinct for the optimization problem. The system will replace the position of existing food sources with the recent sources of food when the recent source position has enhanced quality than the existing food source position. For the well-optimization applications, the premise of $\mathrm{ABC}$ methodology is used and the system attempts to touch the global minimum. The important $\mathrm{ABC}$ parameters and their significant use in this work are given in Table 2.

\begin{tabular}{cc}
\multicolumn{2}{c}{ Table 2. ABC parameters } \\
\hline Parameter & Value \\
\hline Lower bound & 10 \\
Upper bound & 10 \\
Size of Colony & 50 \\
Limit of food source & 100 \\
Max cycle & 1000 \\
\hline
\end{tabular}

Getting stuck in local minima is a major drawback of backpropagation. So, an efficient optimization algorithm like $\mathrm{ABC}$ is used for the optimization and the result is trained with the $\mathrm{BP}$ algorithm. Here, a hybrid ABC-BP method called Modified ABC is introduced.

\subsection{Load scheduling formulation}

To reduce the cost of electric energy, find the optimal combination of sources based on demand. For any unit, the cost curve is supposed by quadratic function segments of the active response of the sources [30]. For a power system, the optimization of cost is defined by the equation:

$$
F_{\text {tot }}=\sum_{i=1}^{n} F\left(P_{i}\right)=\sum_{i=1}^{n}\left(a_{i} P_{i}^{2}+b_{i} P_{i}+c_{i}\right)
$$

Where $F_{\text {tot }}$ is the total cost of generation, $a_{i}, b_{i}$ and $c_{i}$ are the cost coefficients and $P_{i}$ is the generated power by the $i^{\text {th }}$ unit and $\mathrm{n}$ is the number of sources. The optimization of cost is subjected to the inequality constraints of the system:

$$
\text { For } \mathrm{i}=1,2,3 \ldots \ldots \mathrm{n}
$$

where $P_{i, \max }$ and $P_{i, \min }$ are the maximum and minimum power outputs.

$$
\text { The total power demand, } P_{D}=\sum_{i=1}^{n} P_{i}-P_{L}
$$

where $P_{L}$ is the total transmission loss. 


\subsection{Modified ABC algorithm}

$\mathrm{BP}$ algorithm is capable to find local optimistic response and $\mathrm{ABC}$ has to find global optimistic response [31]. In this paper, the hybrid algorithm ABC-BP is modified and is termed as a Modified ABC Optimization algorithm (MABCO). In this hybrid technology, in the initial stage of computation, the optimization technique $\mathrm{ABC}$ is used to find the optimum values from the training parameters, and $\mathrm{BP}$ is continued with this optimized result.

Algorithm 2:

a) Initialize the population by setting the constraints like colony size, heuristic figures, and ending the function.

b) Generate a new solution for employed bees and apply a greedy selection process.

c) Sharing the information between employed and onlooker bees. The evaluation of shared information is carried out by the Onlooker bee and randomly selects a probability-based food source. This is proportional to the food source quality. Calculate the probability using the modified expression

d) Construct and evaluate the fitness functions of all solutions in the population and memorize the best solution.

e) Repeat the process to obtain the optimized values, and then exit.

f) Updating of initial weights of BP will take place when the exit condition become fulfilled

g) Train the BPNN and calculate the mean square error (MSE). The system then goes through the stopping condition.

h) If the system declines the stopping condition then reiterate the system using step 7. Or else, maintain the outcomes for MSE and Regression.

\section{RESULTS AND DISCUSSION}

The simulations have been conducted with different requirements of the power system. The modified scheduling algorithm, MABC runs many times and the average makespan value is computed. In all the training, the optimum solutions are caused before reaching the maximum iteration. The result is compared with the BPNN and ABC-BP algorithm. The obtained value with different ANN structure is shown in Table 3 and Table 4. In Table 3 the ANN structure consists of 6 input layers, 10 hidden layers, and 1 output layers (6-10-1). These systems take 142.11 seconds for the execution and its accuracy is 98.88 . In Table 4 , the number of the hidden layer is changed to fifteen (6-15-1). It results in an improvement in accuracy.

Figure 1 illustrates the best training behavior of the modified algorithm - MABC. The MSE is 1.03930e-12 at Epoch 220 as shown in Table 4. The performance of the system is analyzed using MSE and Epochs. The graph shows the trends in training, validation, and test data in terms of MSE and Epochs. From the graph, it is clear that the MSE decreases with an increase in the number of epochs for all trained, validation, and test data. The accuracy of the system is $99.23 \%$ on the basis of performance parameters.

Table 3. Time, MSE and e pochs for 6-10-1 ANN architecture

\begin{tabular}{cccc}
\hline Algorithm & BPNN & ABC-BP & MABCO \\
\hline CPU TIME & 68.14 & 178.23 & 142.11 \\
EPOCHS & 1000 & 820 & 274 \\
MSE & $6.45 \times 10^{-3}$ & $1.89 \times 10^{-7}$ & $1.88 \times 10^{-12}$ \\
ACCURACY $(\%)$ & 86.12 & 97.61 & 98.88 \\
\hline
\end{tabular}

Table 4. Time, MSE and epochs for 6-15-1 ANN architecture

\begin{tabular}{cccc}
\hline Algorithm & BPNN & ABC-BP & MABCO \\
\hline CPU TIME & 74.25 & 182.23 & 142.88 \\
EPOCHS & 1000 & 812 & 220 \\
MSE & $4.36 \times 10^{-3}$ & $1.45 \times 10^{-7}$ & $1.04 \times 10^{-12}$ \\
ACCURACY $(\%)$ & 88 & 98.11 & 99.23 \\
\hline
\end{tabular}

In this load scheduling approach, a three-layer - input, output, and hidden layers - feed-forward ANNs are used. For the optimization of load, a swarm-based meta-heuristic scheduling algorithm, MABCO is introduced. It advises to minimize the cost of energy, minimize the energy conception during peak time, and make the availability of the power to the highly demanded areas. The electricity demand is not reduced, but it tries to shift the demand from peak to off-peak period by considering all other parameters. The 
traditional load shedding process is completely avoided by using this scheduling process. The consumptions of unscheduled and scheduled load are shown in Figure 2 and Figure 3 respectively.

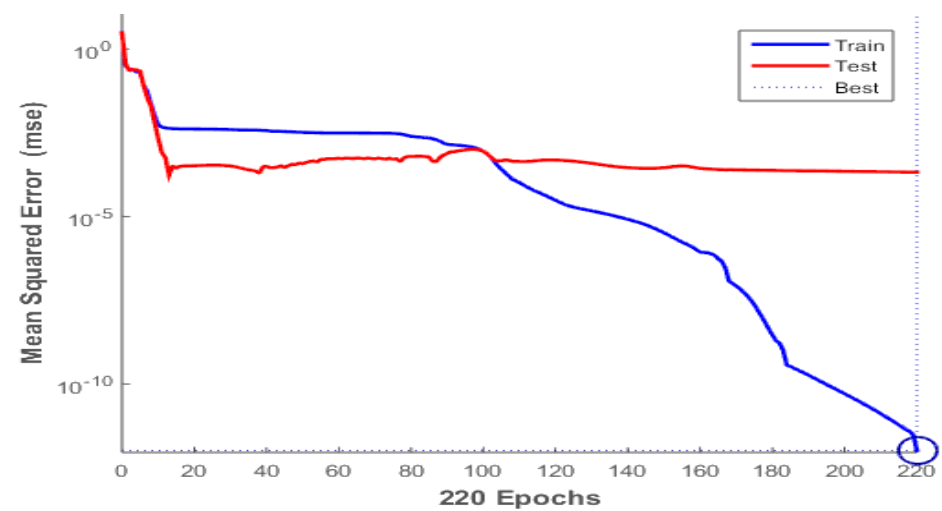

Figure 1. Training performance of MABC-BP

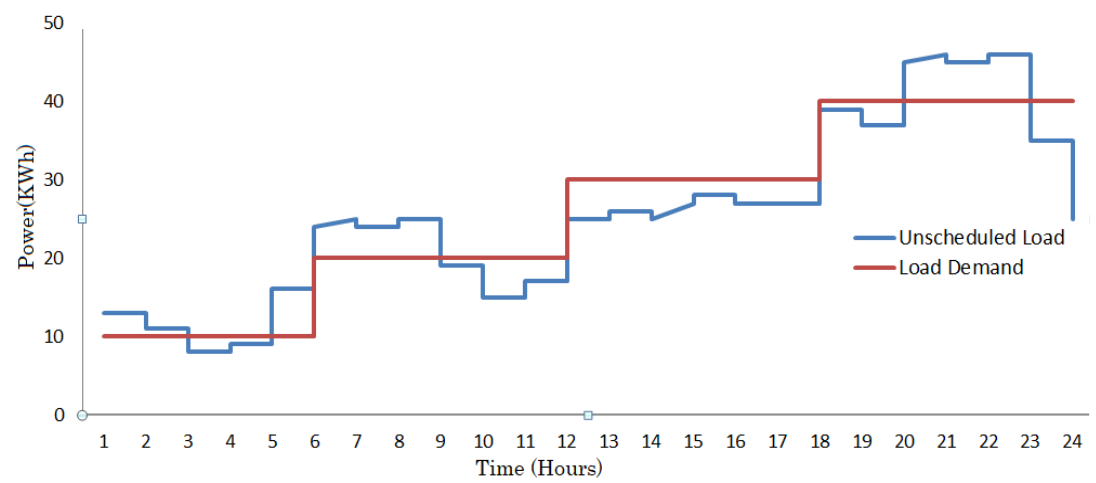

Figure 2. Unscheduled power consumption

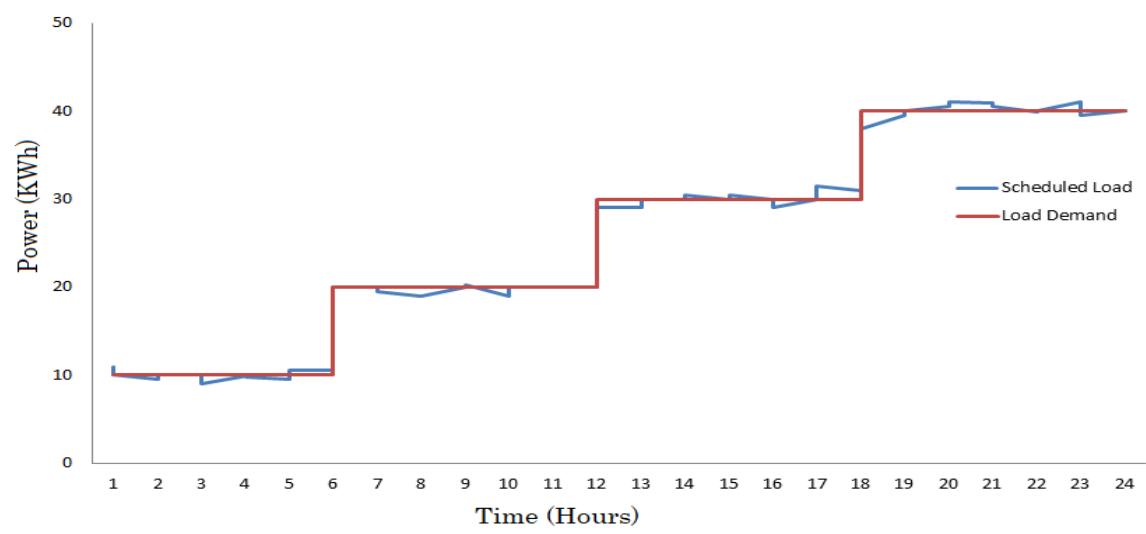

Figure 3. Scheduled power consumption

The scheduled power system minimizes the error. It also reduces the cost of energy and makes the availability to meet the demanded load. Before the scheduling process, $6.7 \%$ of additional energy is delivered, but after the scheduling, it reduces to $0.8 \%$. After scheduling the essential energy to meet the demanded load is reduced from $8.04 \%$ to $0.96 \%$. Figure 4 (a) shows the percentage of extra generated power for both scheduled and unscheduled systems. Figure 4(b) shows the percentage of power needed to meet the demanded load in both systems. 


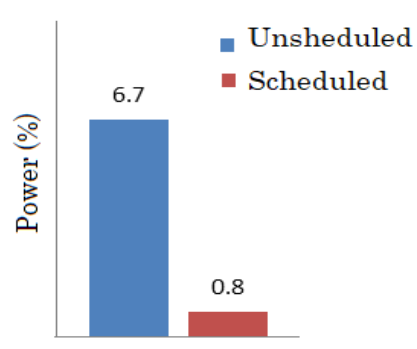

(a)

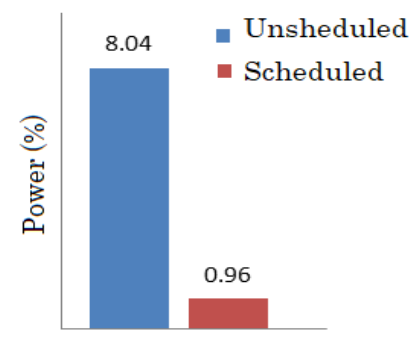

(b)

Figure 4. (a) Extra energy and (b) Essential energy for the unscheduled and scheduled system

\section{CONCLUSION}

In this paper, the MABC has been introduced for optimal scheduling. The BPNN training algorithm is used for optimizing of the feed-forward neural network. Slow speed and local minimization are some drawbacks of this algorithm. Therefore, some meta-heuristic optimization algorithms inspired by nature are used to solve the sophisticated problems. In this work, the MABC is used to train the BPNN. Initially, to train the network MABC algorithm is used, then the learning process is advancing with BPNN by accepting the finest weight sets from the optimized algorithm. It minimizes the error and gives a better accuracy rate. The performance is compared with other existing algorithms like BPNN and ABC-BP. The scheduled system has better performance and it helps to minimize the energy utilization without affecting the demanded power. It also helps to reduce energy costs. The experimental results indicate that this hybrid algorithm performed better than its individual implementations. Therefore, it is concluded that the present system can produce a better optimal schedule efficiently.

\section{REFERENCES}

[1] V. Moorthy, et al., "Investigation on the effectiveness of ABC algorithm for hydrothermal energy management considering emission aspects," International Journal of Energy Sector Management, vol. 9, pp. 251-273, 2015.

[2] Kabalci, et al.,"A modified ABC algorithm approach for power system harmonic estimation problems," Electric Power Systems Research, vol. 154, pp. 160-173, 2018.

[3] Dieu, V.N. and Ongsakul, W, "Improved merit order and augmented Lagrange Hopfield network for short term hydrothermal scheduling," Energy Conversion and Management, vol. 50, no. 12, pp. 3015-3023, 2009.

[4] A. I. Selvakumar, "Civilized swarm optimization for multi-objective short-term hydrothermal scheduling," Electrical Power and Energy Systems, vol. 51, pp. 178-189, 2013.

[5] Basheer, I.A., and Hajmeer, "Artificial neural networks: fundamentals, computing, design, and application," Journal of Microbiological Methods, vol. 43, no. 1, pp. 3-31, 2000.

[6] Mohd Nawi, et al., "An accelerated particle swarm optimization based levenberg marquardt back propagation algorithm,". In: Loo C.K., et al.,(eds), Neural Information Processing. ICONIP 2014. Lecture Notes in Computer Science, Springer, Cham, vol. 8835, pp. 245-253, 2014.

[7] F. S. Abu-Mouti and M. E. El-Hawary, "Overview of Artificial Bee Colony (ABC) algorithm and its applications," 2012 IEEE International Systems Conference, Vancouver, pp. 1-6, 2012.

[8] Hota, et al., " Short-term hydrothermal scheduling through evolutionary programming technique," Electric Power Systems Research, vol. 52, no. 2, pp.189-196, 1999.

[9] Sifuentes, et al., "Hydrothermal scheduling using benders decomposition: accelerating techniques," IEEE Transactions on Power Systems, vol. 22 no. 3, pp. 1351-1359, 2007.

[10] B. Mousumi, "Hopfield neural networks for optimal scheduling of fixed head hydrothermal power systems," Electric Power Systems Research, vol. 64, no. 1, pp. 11-15, 2003.

[11] K. C. Le, et al."Environmental economic hydrothermal system dispatch by using a novel differential evolution," Journal of Engineering and Technological Sciences, vol. 50, no. 1, pp. 1-20, 2018.

[12] Chao-Lung Chiang, "Optimal economic emission dispatch of hydrothermal power systems," International Journal of Electrical Power \& Energy Systems, vol. 29, no. 6, pp. 462-469, 2007.

[13] Zhai Rongrong, et al., "The Optimization of Power Dispatch for Hydro-thermal Power Systems," Procedia Environmental Sciences, vol. 11, part B, pp. 624-630, 2011.

[14] Chen, X., et al., "A Two-Stage strategy to handle equality constraints in ABC-based power economic dispatch problems," Soft Computing. doi:10.1007/s00500-018-03723-4, vol. 23, no. 15, pp. 6679-6696, 2019.

[15] S. Y. W.Wong. "Hybrid simulated annealing/genetic algorithm approach to short-term hydro-thermal scheduling with multiple thermal plants," Electrical Power and Energy Systems, vol. 23, no. 7, pp. 565-575, 2001.

[16] D. Karaboga and A. Bahriye, "A comparative study of artificial bee colony algorithm," Applied Mathematics and Computation, vol. 214, no. 1, pp. 108-132, 2009. 
[17] K. Lenin and B. Ravindhranath Reddy, "Voltage profile enhancement and reduction of real power loss by hybrid biogeography based artificial bee colony algorithm," Indonesian Journal of Electrical Engineering and Informatics (IJEEI), vol. 2, no. 2, pp. 86-95, 2014.

[18] Rashid, et al., "A hybrid of artificial bee colony, genetic algorithm, and neural network for diabetic mellitus diagnosing," ARO-The Scientific Journal of Koya University, vol. 6, no. 1, pp. 55-64, 2018.

[19] C. S. Rao, "Design of artificial intelligent controller for automatic generation control of two area hydrothermal system," International Journal of Electrical and Computer Engineering (IJECE), vol. 2, no. 2, pp. 183, 2012.

[20] A. Suliman and Y. Zhang, "A Review on Back-Propagation Neural Networks in the Application of Remote Sensing Image Classification,” Journal of Earth Science and Engineering, vol. 5, pp. 52-65, 2015.

[21] M. Alsmadi., et al., "Back propagation algorithm: the best algorithm among the multi-layer perceptron algorithm," International Journal of Computer Science and Network Security, vol. 9, pp. 378-383, 2009.

[22] D. Graupe. "Principles of Artificial Neural Networks," Advanced Series in Circuits and Systems, Singapore, Hackensack, N. J.: World Scientific, vol. 7, no. 3, 2013.

[23] Vijo M Joy and S Krishnakumar, "Efficient load scheduling method for power management," International Journal Of Scientific \& Technology Research, vol. 5, no. 1, pp. 99-101, 2016

[24] D. Karaboga and B. Basturk, "On the performance of artificial bee colony (ABC) algorithm," Applied Soft Computing, vol.8, no. 1, pp. 687-697, 2008.

[25] A. A. Seker and M. H. Hocaoglu, "Artificial bee colony algorithm for optimal placement and sizing of distributed generation," 8th International Conference on Electrical and Electronics Engineering, 2013, pp. 127-131.

[26] D. Karaboga and B. Akay, "A modified Artificial Bee Colony (ABC) algorithm for constrained optimization problems," Applied Soft Computing, vol. 11, no. 3, pp. 3021-3031, 2011

[27] W. Gao, et al., "A global best artificial bee colony algorithm for global optimization,” Journal of Computational and Applied Mathematics, vol. 236, no. 11, pp. 2741-2753, 2012.

[28] Dervis Karaboga and Bahriye Akay, "A comparative study of artificial bee colony algorithm," Applied Mathematics \& Computation, vol. 214, no. 1, pp.108-132, 2009.

[29] Karaboga, D. and Basturk, B., "A powerful and efficient algorithm for numerical function optimization: artificial bee colony (ABC) algorithm," Journal of Global Optimization, vol. 39, no. 3, pp. 459-471, 2007.

[30] H. Hardiansyah, "Artificial Bee Colony Algorithm for Economic Load Dispatch Problem," IAES International Journal of Artificial Intelligence (IJAI), vol. 2, no. 2, pp. 90-98, 2013.

[31] C. Ozturk, and D. Karaboga. "Hybrid artificial bee colony algorithm for neural network training," IEEE Congress of Evolutionary Computation (CEC), pp.84-88, 2011.

\section{BIOGRAPHY OF AUTHORS}

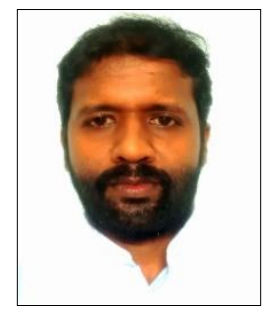

Vijo M Joy received his Master's degree in Electronics in 2006 from Mahatma Gandhi Univerisity, Kottayam, Kerala. Completed his M. Tech in Electronics with a specialization in Embedded System Design from IGNOU in 2011. He working as faculty and Head of the Department of Electronics, Aquinas College, Edacochin, Kochi, India and is pursuing his research in Electronics at School of Technology \& Applied Sciences, Mahatma Gandhi Univerisity Regional Center, Edappally, Kochi. His current areas of research include Artificial Intelligence and its applications. He served as a reviewer of indexed international journals including Advances in Science, Technology and Engineering Systems Journal, and American Journal of Neural Networks and Applications. He has ten years of teaching experience.

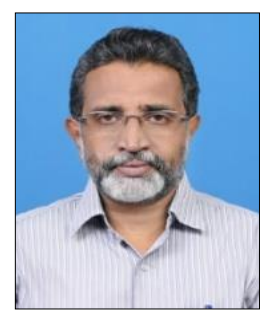

Dr. S Krishnakumar is a doctoral degree holder in Thin Film Devices from Mahatma Gandhi University, Kottayam, Kerala, India in 1995. Completed his M.Tech in Computer Science from the Sam Higginbottom University of Agriculture, Technology and Sciences, formerly Allahabad Agricultural Institute, in 2006 and also got MCA from IGNOU in 2010. From 2013 to 2017 he worked as the Regional Director at the School of Technology and Applied Sciences, Cochin. Presently he is a research guide in Electronics at Mahatma Gandhi University. His current area of research activities is in Artificial Intelligence, Circuit Design, and Image processing. He has twenty-four years of teaching experience and an Associate Member of the Institute of Engineers, India. He served as a member of the Academic council of M G University, Kottayam and a member of the Board of studies of Calicut University. 